\title{
Decision Analysis: die Stärke des Internisten
}

\author{
R. Schoenenberger
}

Eine 79jährige Frau mit einer stabilen koronaren Herzkrankheit wird hospitalisiert zur Abklärung von Fieber und Gewichtsverlust. Anamnese und klinischer Status sind diagnostisch unergiebig. Im Labor finden sich eine normochrome, normozytäre Anämie von 8,2 g\% und eine auf das Doppelte der Norm erhöhte LDH-Aktivität. Im thorako-abdominalen CT sieht man grosse mesenteriale Lymphknotenpakete. Sie vermuten das Vorliegen eines malignen Lymphoms. Eine Knochenmarkspunktion und -biopsie ergeben keine definitive Diagnose. Wie weiter?

Korrespondenz:

PD Dr. med. Ronald Schoenenberger Bürgerspital

CH-4500 Solothurn
Probleme wie diese stellen sich in der Klinik und Praxis des Internisten fast täglich. Es taucht eine Vielzahl von Fragen auf. Was ist die Lebenserwartung einer 79jährigen Frau ohne malignes Lymphom? Auf welchem Wege kann eine histologische Lymphomdiagnose in dieser Situation am besten erzwungen werden? Muss sie erzwungen werden? Was ist die Morbidität und Mortalität einer laparoskopischen Biopsie von Lymphknoten im Mesenterium? Gibt es Therapieoptionen, mit denen einer solchen Patientin ein längeres Leben mit guter Qualität ermöglicht werden kann? Was will die Patientin? Wie stellt sie sich ihr Lebensende vor? Was für Risiken ist sie bereit einzugehen? Und zu guter Letzt: Was kostet die weitere Diagnostik und eventuell Therapie dieses wahrscheinlich malignen Lymphoms?

Es müssen nicht immer sogenannt grosse Entscheide sein, wie zum Beispiel, ob bei einem über 90jährigen Patienten noch eine Aortenklappenersatzoperation durchgeführt werden soll. Auch kleinere medizinische Entscheidungen, beispielsweise welche radiologische Technik zur Diagnose einer Spinalkanalstenose angewandt werden soll, beinhalten drei wesentliche Elemente, die jedem Entscheidungsprozess innewohnen: 1. Ein Entscheid muss gefällt werden! 2. Die Konsequenzen der Entscheidung sind nie zu 100\% sicher und klar; 3. von allen am Entscheidungsprozess direkt oder indirekt beteiligten Personen oder Institutionen haben sicher zwei Mitspieler divergierende Interessen und Ziele (welcher Spitaldirektor befürwortet schon die Durchführung auswärtiger MRI-Untersuchungen?).

Im medizinischen Entscheidungsprozess in Situationen wie der oben dargelegten ist sicher der breit ausgebildete und erfahrene Internist gefragt, welcher mit Hilfe der fundierten Empirie unter Berücksichtigung der klinischen Gesamtsituation mit einer guten Portion Intuition (die Kunst der Medizin) einen Entscheid auch bei - eingestandener oder uneingestandener grosser Ungewissheit fällen wird. Wer ist nicht schon froh gewesen um eine solche Internistin oder einen solchen Internisten?

Leider sind aber solche Entscheidungsträger und -finder immer seltener zu finden. Bedingt durch die Vielzahl der medizinisch-technologischen Möglichkeiten und den immer grösser werdenden Stand des Wissens in Detailaspekten wird es immer schwieriger, bei medizinischen Entscheidungen objektiv, vollständig und gerecht zu bleiben. Hinzu kommt der zunehmende Individualismus in unserer Gesellschaft, mit dem ein Entscheid für einen Patienten nicht für einen anderen in der gleichen Situation gelten kann. Soll dann noch der zunehmende Druck, beschränkte finanzielle Ressourcen effizient einzusetzen, berücksichtigt werden, so braucht es schon sehr viel Erfahrung und auch sehr viel Intuition, um schwierige Entscheide manchmal sehr rasch zu fällen. Und entschieden werden muss!

In dieser Situation kann die Decision Analysis Hilfestellungen bieten. Ursprünglich von Ökonomen und Politikwissenschaftlern entwickelt, bietet die medizinische Variante eine Vielzahl von Techniken an, mit denen Entscheidungsprozesse sozusagen seziert werden können. Entscheide werden in Einzelschritte zerlegt und dann Verzweigungen mit Wahrscheinlichkeitsdaten aus der klinischen Epidemiologie versehen. Die Ansichten der betroffenen Patienten und auch ökonomische Daten können integriert werden. Es entstehen Entscheidungsbäume, die mit erstaunlich viel Leben gefüllt sein können, obwohl sie so trockene Namen wie Kosten-Nutzen-Analyse, Kosten-Effektivitäts-Analyse oder Markov-Modelle tragen.

Die Techniken der klinischen Decision Analysis sind verstehbar, ja sogar erlernbar. Am diesjährigen Jahreskongress der Schweizerischen Gesellschaft für Innere Medizin werden drei prominente Experten - Prof. A. Perrier (Genf), Prof. T. Thomson (Newcastle, UK) und Prof. J. Steurer (Zürich) - in einem Seminar an praktischen Beispielen die Instrumente der klinischen Decision Analysis auf die Bedürfnisse von Internistinnen und Internisten zugeschnitten darlegen. Ich glaube, dass man als Entscheider von diesem Seminar sehr viel wird profitieren können. Man wird lernen können, dass die klinische Decision 
Analysis nicht automatisch Antworten liefert, aber als Rahmen und Netzwerk dienen kann, in dem man klinische Probleme effektiver und möglicherweise gerechter lösen kann. Die klinische Decision Analysis ersetzt Erfahrung nicht, aber sie kann Erfahrung ergänzen. Bei der Decision Analysis wird von der Internistin und vom Internisten verlangt, das, was er oder sie sowieso tut, etwas systematischer zu tun. So können jüngere und weniger erfahrene Internistinnen und Internisten lernen. Patienten können ihre Präfe- renzen besser einfliessen lassen. Und die Verantwortlichen für die Ressourcenverteilung können sehen, dass Verschwendung keinen wesentlichen Grund für die Kostenentwicklung in der Medizin darstellt.

(Bei der eingangs erwähnten Patientin wurde ein malignes grosszelliges B-Zell-Lymphom aus einer laparoskopischen Biopsie diagnostiziert und eine Chemotherapie mit CHOP-Mapthera vorgeschlagen.)

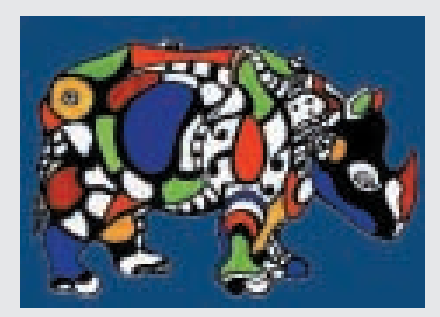

\author{
71. Jahresversammlung \\ der Schweizerischen Gesellschaft für Innere Medizin \\ sowie Jahrestagung der \\ Schweizerischen Gesellschaft für Hämatologie \\ Schweizerischen Fachgesellschaft für Geriatrie \\ Sektion Klinische Pharmakologie und Toxikologie \\ Schweizerischen Hypertonie Gesellschaft
}

Ort und Datum: Basel, Mittwoch, 21. Mai, bis Freitag, 23. Mai 2003

Der Klinische Track soll Gelegenheit geben, nicht nur das Neueste, sondern das Entscheidendste zu erfahren.

Clinical year in review: Exponenten aus verschiedenen Fachgebieten der Medizin bieten einen Überblick über neueste wissenschaftliche und klinisch relevante Erkenntnisse ihres Faches.

Clinical pearls: Chefärzte der Nordwestschweiz präsentieren ausgewählte Fälle.

Workshops: Grundversorger und Experten bearbeiten gemeinsam mit dem Publikum ein Thema in kleinen Gruppen.

Skill lab: Informatik, Blutdruckmessung, Gastroskopie, Bronchoskopie, Inhalationstechniken, Prostatapalpation, Selbststudiumprogramme in Radiologie, Ergonomie am Arbeitsplatz.

Sachverstandprüfung im Strahlenschutz BAG: 21. Mai, 11.45 Uhr.

Administratives Sekretariat: Jahresversammlung SGIM 2003, c/o AKM Congress Service, Postfach, 4005 Basel, Tel. 06168677 11, Fax 06168677 88, E-Mail: info@akm.ch

Website mit weiteren Infos: www.akm.ch/sgim2003 